\title{
Prognostic value of chronic hepatitis $B$ virus infection in patients with breast cancer in a hepatitis B virus endemic area
}

\author{
Weikai Xiao ${ }^{1,2 \#}$, Ying Zhou ${ }^{3 \#}$, Ping Yu ${ }^{4 \#}$, Anli Yang ${ }^{1}$, Shaoquan Zheng ${ }^{1}$, Hailin Tang ${ }^{1}$, Xiaoming Xie ${ }^{1}$ \\ ${ }^{1}$ Department of Breast Oncology, Sun Yat-sen University Cancer Center, State Key Laboratory of Oncology in South China, Collaborative \\ Innovation Center for Cancer Medicine, Guangzhou 510060, China; ${ }^{2}$ Department of Breast Cancer, Cancer Center, Guangdong Provincial People's \\ Hospital, Guangdong Academy of Medical Sciences, Guangzhou 510080, China; ${ }^{3}$ Haizhu District Center for Disease Control and Prevention, \\ Guangzhou 510288, China; ${ }^{4}$ Department of Anesthesiology, Sun Yat-sen University Cancer Center, State Key Laboratory of Oncology in South \\ China, Collaborative Innovation Center for Cancer Medicine, Guangzhou 510060, China \\ Contributions: (I) Conception and design: W Xiao, X Xie, H Tang; (II) Administrative support: None; (III) Provision of study materials or patients: W \\ Xiao, A Yang, S Zheng; (IV) Collection and assembly of data: Y Zhou, P Yu; (V) Data analysis and interpretation: W Xiao, Y Zhou; (VI) Manuscript \\ writing: All authors; (VII) Final approval of manuscript: All authors. \\ "These authors contributed equally to this work. \\ Correspondence to: Xiaoming Xie, MD, PhD; Hailin Tang, MD, PhD. Department of Breast Oncology, Sun Yat-sen University Cancer Center, State \\ Key Laboratory of Oncology in South China, Collaborative Innovation Center for Cancer Medicine, 651 Dongfeng East Road, Guangzhou 510060, \\ China. Email: xiexm@sysucc.org.cn; tanghl@sysucc.org.cn.
}

Background: Except for hepatocellular carcinoma, chronic hepatitis B virus (HBV) infection has also been reported to be associated with increased morbidity and mortality of other cancers. However, the impact of chronic HBV infection on the prognosis of breast cancer (BC) remains unclear. Our study aimed to evaluate the prognostic value of $\mathrm{HBV}$ infection for $\mathrm{BC}$ in an endemic area of $\mathrm{HBV}$ in China.

Methods: There was a total of 1,904 patients with early BC who underwent mastectomy or breastconserving surgery enrolled in our study. HBV infection on overall survival (OS) and hepatic metastasis-free survival (HMFS) was the main research indicator for this study.

Results: A total of 212 patients (11.1\%) were identified with chronic HBV infection due to serum hepatitis B surface antigen (HBsAg) positive. HBsAg-positive patients had inferior OS (84.9\% vs. 90.4\%, $\mathrm{P}=0.005)$ and HMFS (92.5\% vs. 97.1\%, $\mathrm{P}=0.016)$ at 5 years than HBsAg-negative patients. Chronic HBV infection was an independent predictor of poor OS in patients with BC [multivariate analysis; hazard ratio (HR), 1.52; $\mathrm{P}=0.038$, but not for HMFS. Subgroup analysis showed that chronic HBV infection was an unfavorable independent prognostic factor for OS in patients with stage II/III BC (HR, 1.59; P=0.025). The 5-year OS and HMFS rates of HBsAg-positive patients were $81.9 \%$ and $90.5 \%$ for patients with stage II/III BC, while those rates of HBsAg-negative patients were $88.5 \%$ and $96.3 \%$, respectively. In stage I patients, there was no significant difference in 5 -year OS (95.8\% vs. 97.1\%; $\mathrm{P}=0.629)$ and HMFS (100.0\% vs. 99.0\%; $\mathrm{P}=0.447)$.

Conclusions: In conclusion, chronic HBV infection predicts a worse prognosis in patients with stage II/III BC, but not stage I BC.

Keywords: Breast cancer (BC); hepatitis B virus infection (HBV infection); clinic-pathological features; survival; liver metastasis

Submitted Sep 26, 2019. Accepted for publication Jan 13, 2020.

doi: 10.21037/atm.2020.01.97

View this article at: http://dx.doi.org/10.21037/atm.2020.01.97 


\section{Introduction}

Chronic hepatitis B virus (HBV) infection has been recognized as an urgent public health problem due to high infection rates, with more than 240 million chronic HBV carriers worldwide (1). China is one of the countries with a high incidence of hepatitis B infection in the world, while South China is one of the regions with the highest rate of chronic HBV infection in China. The seroprevalence of hepatitis B surface antigen (HBsAg) is $10 \%$ to $12 \%$ in the general population in South China (2). Chronic HBV infection has been widely confirmed as a causative factor in chronic hepatitis, cirrhosis and hepatocellular carcinoma (HCC). However, in addition to HCC, it has also been reported that chronic $\mathrm{HBV}$ infection affects the progression of other tumors, including non-Hodgkin lymphoma $(3,4)$, hepatic cholangiocarcinoma $(5,6)$, leukemia $(7)$, gastric cancer (8), nasopharyngeal cancer (9), and pancreatic cancer (10). For example, HBV-infected non-HCC cancer patients, such as nasopharyngeal (9) or pancreatic cancer (11), have significantly worse clinicopathological features and prognosis than uninfected patients.

However, the impact of chronic HBV infection on the clinicopathological features and prognosis of patients with breast cancer (BC) is unclear. Therefore, this study intends to investigate the impact of chronic $\mathrm{HBV}$ infection on the clinicopathological features and prognosis of patients with $\mathrm{BC}$ in an epidemic area of HBV.

\section{Methods}

\section{Study population and data extraction}

This study mainly retrospectively collected patients with BC who had undergone surgery at the Sun Yat-sen University Cancer Center (Guangzhou, China) from February 2008 to December 2010. A total of 1,904 patients with BC who were pathologically confirmed and had no distant metastasis were identified. Patients with missing basic information such as tumor staging and unknown follow-up were excluded. All patients signed the informed consent form, and the study was approved by the Ethics Committee of the Cancer Center of Sun Yat-sen University. The staging of the tumor was performed according to the 7th edition of the TNM staging system of the American Joint Committee on Cancer (AJCC).

\section{Serological detection of HBV infection}

Blood tests for HBV infection in this study were performed before surgery for BC. Briefly, HBV is detected by collecting blood samples, separating serum, and then measuring serum samples by enzyme-linked immunosorbent assay. To ensure the accuracy of the test, HBV testing is performed and quality controlled according to standard operating procedures.

\section{Patient follow-up and statistical analysis}

All patients were routinely followed up after surgery. Patients were followed up every 3 months for the first 2 years, and every 3 to 6 months for the 3 rd to 5 th years, and 1-2 times a year until the death after the 5 th year. The duration of follow-up refers to the interval between the diagnosis of $\mathrm{BC}$ to death or the last follow-up. The median follow-up time for HBsAg-positive patients was 68.5 months, compared with 70 months for HBsAgnegative patients. The effect of chronic $\mathrm{HBV}$ infection on overall survival (OS) and hepatic metastasis-free survival (HMFS) was the main research indicator for this study. We calculated the interval from the first day of diagnosis to the death or the last follow-up as OS and calculated the interval from the first day of diagnosis to the clinical detection of liver metastases as HMFS.

SPSS software (version 21.0; SPSS Inc., Chicago, IL, USA) was adopted to perform most of the statistical analysis. Chi-square test was used to compare statistical differences in clinical and pathological variables between HBsAg-positive and HBsAg-negative patients. When we analyzed the effect of chronic HBV infection on patient survival, we used KaplanMeier survival analysis to plot the survival curve and the difference was compared using a log-rank test. Statistically significant variables after univariate analysis were further used in the multivariate analysis of the Cox proportional hazard model to test the independent significance of the variables. The standard for statistical significance is set to 0.05 , and all $\mathrm{P}$ values are based on two-sided testing. Kaplan-Meier curves for OS and HMFS were plotted by SAS software (SAS Institute Inc. version 9.4, USA).

\section{Results}

A total of $212(11.1 \%)$ of the 1,904 patients were seropositive HBsAg. $\mathrm{HBsAg}$-positive and $\mathrm{HBsAg}$-negative patients are similar in most clinicopathological features. Besides, there was no significant difference in the surgical approach between the two groups. However, the proportion of younger patients (age $\leq 35$ years) in the HBsAg-positive 
group was higher $(15.6 \%$ vs. $9.0 \% ; \mathrm{P}=0.003)$ compared with the HBsAg-negative group in patients with BC (Table 1). In addition, the premenopausal patients in the HBsAgpositive group also had a higher proportion than the HBsAg-negative group ( $70.8 \%$ vs. $61.2 \% ; \mathrm{P}=0.004)$ (Table 1). Finally, the percentage of patients with lymphovascular invasion in the HBsAg-positive group was significantly higher than that in the HBsAg-negative group (5.2\% vs. $2.5 \% ; \mathrm{P}=0.042$ ) (Table 1 ).

Effect of chronic HBV infection on the prognosis of patients with $B C$

The 5 -year OS rate $(84.9 \%$ vs. $90.4 \%, \mathrm{P}=0.005)$ was significantly lower in HBsAg-positive BC patients than in HBsAg-negative patients (Figure 1A). Univariate analysis showed that the OS of HBsAg-positive BC patients was significantly worse than HBsAg-negative patients (Table 2). To adjust the influence of various confounding factors, Cox proportional hazards regression model is used for multivariate analysis. Multivariate analysis further determined that, chronic HBV infection is an independent risk factor for OS in patients with $\mathrm{BC}$ [hazard ratio (HR), 1.52; $95 \%$ confidence interval (CI), 1.02-2.26, $\mathrm{P}=0.038$ ] (Table 2). Furthermore, the 5 -year HMFS $(92.5 \%$ vs. $97.1 \%$, $\mathrm{P}=0.016)$ of patients with chronic HBV infection were significantly shorter compared with those without HBV infection (Figure $1 B$ ). In addition, later $\mathrm{T}$ and $\mathrm{N}$ staging were also independent risk factors for poor prognosis (Table 2).

Effect of chronic HBV infection on survival outcome in BC patients with luminal or non-luminal $B C$

In patients with luminal $\mathrm{BC}, \mathrm{HBsAg}$-positive patients had worse OS compared with HBsAg-negative patients $(85.7 \%$ vs. 91.7\%; $\mathrm{P}=0.016$ ) (Figure $2 A$ ). Multivariate analysis further confirmed that chronic HBV infection was an independent prognostic factor for OS in patients with luminal BC (HR, 1.62; $95 \%$ CI, $1.03-2.55$; $\mathrm{P}=0.038$ ). However, there is no significant association between chronic $\mathrm{HBV}$ infection and HMFS in luminal BC patients (Figure $2 B$ ). In non-luminal BC patients, chronic HBV infection also appeared to be associated with worse OS, but no statistical difference was observed (79.0\% vs. 84.9\%; $\mathrm{P}=0.139$ ) (Figure 2C). Moreover, chronic $\mathrm{HBV}$ infection was significantly associated with poor 5-year HMFS (82.9\% vs. 95.8\%; $\mathrm{P}=0.002$ ) (Figure 2D) in patients with non-luminal BC.
Table 1 Baseline characteristics of HBsAg-positive and HBsAg-negative BC patients

\begin{tabular}{|c|c|c|c|}
\hline Variable & $\begin{array}{c}\mathrm{HBsAg}^{+}(\%) \\
(\mathrm{N}=212)\end{array}$ & $\begin{array}{c}\mathrm{HBsAg}^{-}(\%) \\
(\mathrm{N}=1,692)\end{array}$ & $P$ value \\
\hline Age at diagnosis & & & 0.003 \\
\hline$\leq 35$ years & $33(15.6)$ & $153(9.0)$ & \\
\hline$>35$ years & $179(84.4)$ & $1,539(91.0)$ & \\
\hline ER & & & 0.211 \\
\hline Positive & $143(67.5)$ & $1,191(70.4)$ & \\
\hline Negative & $69(32.5)$ & $501(29.6)$ & \\
\hline PR & & & 0.282 \\
\hline Positive & $145(68.4)$ & $1,194(70.6)$ & \\
\hline Negative & $67(31.6)$ & $498(29.4)$ & \\
\hline HER2 & & & 0.379 \\
\hline Positive & $39(19.3)$ & $327(20.6)$ & \\
\hline Negative & $163(80.7)$ & $1,264(79.4)$ & \\
\hline Lymphovascular invasion & & & 0.042 \\
\hline Yes & $11(5.2)$ & $42(2.5)$ & \\
\hline No & $201(94.8)$ & $1,650(97.5)$ & \\
\hline Tumor size & & & 0.291 \\
\hline T1 & $74(34.9)$ & $677(40.0)$ & \\
\hline T2 & $111(52.4)$ & $861(50.9)$ & \\
\hline T3 & $13(6.1)$ & $81(4.8)$ & \\
\hline $\mathrm{T} 4$ & $14(6.6)$ & $73(4.3)$ & \\
\hline Lymph node metastasis & & & 0.286 \\
\hline No & $95(44.8)$ & $888(52.5)$ & \\
\hline N1 & $59(27.8)$ & $406(24.0)$ & \\
\hline N2 & $33(15.6)$ & $238(14.1)$ & \\
\hline N3 & $25(11.8)$ & $160(9.5)$ & \\
\hline Menopause at diagnosis & & & 0.004 \\
\hline No & $150(70.8)$ & $1,035(61.2)$ & \\
\hline Yes & $62(29.2)$ & $657(38.8)$ & \\
\hline Histological grade & & & 0.148 \\
\hline G1 or G2 & $151(71.2)$ & $1,266(74.8)$ & \\
\hline G3 & $61(28.8)$ & $426(25.2)$ & \\
\hline Surgery types & & & 0.075 \\
\hline Mastectomy & $190(89.6)$ & $1,569(92.7)$ & \\
\hline Breast-conserving & $22(10.4)$ & $123(7.3)$ & \\
\hline
\end{tabular}

HBsAg, hepatitis B surface antigen; BC, breast cancer; ER, estrogen receptor; $\mathrm{PR}$, progesterone receptor. 

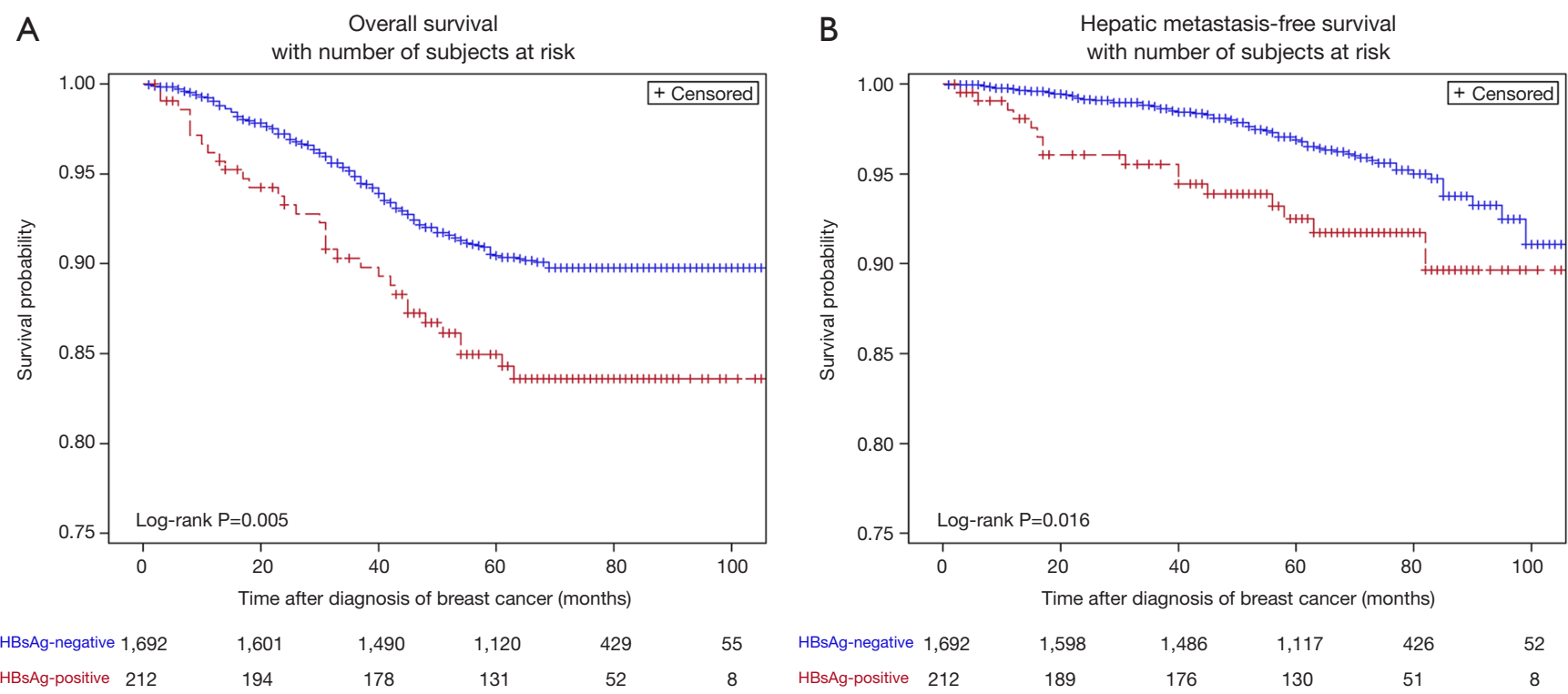

Figure 1 Kaplan-Meier survival curves showing overall survival (A) and liver metastasis-free survival (B) in patients with hepatitis B surface antigen (HBsAg) positive and HBsAg-negative breast cancer. P values were calculated using an unadjusted log-rank test. 95\% CI, 95\% confidence interval.

\section{Effect of chronic HBV infection on survival outcome in BC patients with stage I or II/III}

In stage I patients, no significant difference was observed in OS $(95.8 \%$ vs. $97.1 \% ; \mathrm{P}=0.629$ ) (Figure $3 A$ ) between $\mathrm{HBsAg}$-positive patients and HBsAg-negative patients. In patients with stage II/III BC, HBsAg-positive patients had worse OS compared with HBsAg-negative patients ( $81.9 \%$ vs. $88.5 \% ; \mathrm{P}=0.006$ ) (Figure $3 B$ ). Multivariate analysis further confirmed that chronic HBV infection was an independent prognostic factor for OS in patients with luminal BC (HR, 1.59; 95\% CI, 1.06-2.39; P=0.025) (Table 3). Additionally, chronic $\mathrm{HBV}$ infection predicted a poor 5-year HMFS in stage II/III patients ( $90.5 \%$ vs. $96.3 \%$; $\mathrm{P}=0.016)$ (Figure 3C), but not in patients with stage I BC (Figure 3D).

\section{Discussion}

To the best of our knowledge, the current study is the first large-scale study to determine the impact of chronic HBV infection in an endemic $\mathrm{HBV}$ region on the prognosis of patients with non-metastatic BC. The main finding of this study is that chronic HBV infection is an independent prognostic factor for stage II/III BC, but not stage I BC. In this cohort, the HBsAg positive rate of $\mathrm{BC}$ patients was $11.1 \%$, and this infection rate was basically consistent with the general population in South China. We observed that young $\mathrm{BC}$ patients (less than or equal to 35 years old) accounted for a higher proportion of patients with chronic HBV infection than those older than 35 years. Furthermore, the proportion of premenopausal patients with chronic $\mathrm{HBV}$ infection is also higher in patients with $\mathrm{BC}$ than those without $\mathrm{HBV}$ infection. Another interesting finding is that patients with HBsAg-positive patients have a higher proportion of patients with lymphovascular invasion.

HBV mainly infects the liver and causes necrosis and inflammation of liver cells. In recent years, the impact of chronic HBV infection on cancer patients has received increasing attention. Previous studies have focused on the impact of HBV reactivation on cancer patients (12-15). In recent years, more and more studies have shown that chronic HBV infection can affect the prognosis of nonHCC cancer patients. Wang et al. found that compared with HBsAg-negative patients, diffuse large B-cell lymphoma patients with HBsAg-positive had a later clinical stage at the time of initial diagnosis (16). Liu et al. reported that chronic $\mathrm{HBV}$ infection was an independent risk factor for the survival of patients with locally advanced nasopharyngeal carcinoma (17). Wei et al. found that patients with HBVinfected pancreatic cancer had a worse prognosis and 
Table 2 Multivariate analysis of prognostic factors in patients with BC $(\mathrm{N}=1,904)$

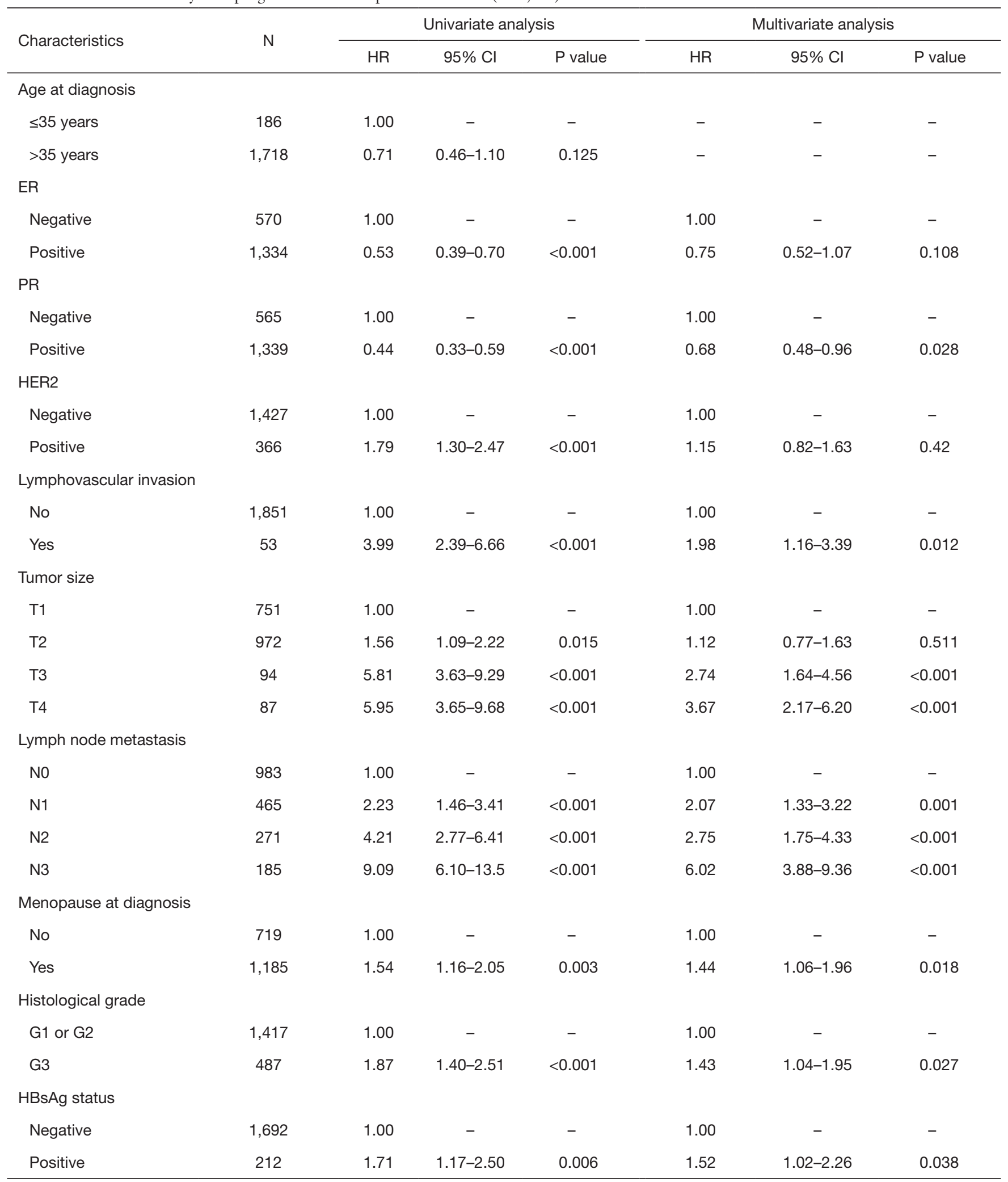

$\mathrm{BC}$, breast cancer; HR, hazard ratio; Cl, confidence interval; ER, estrogen receptor; PR, progesterone receptor; HBsAg, hepatitis B surface antigen. 
A

Overall survival

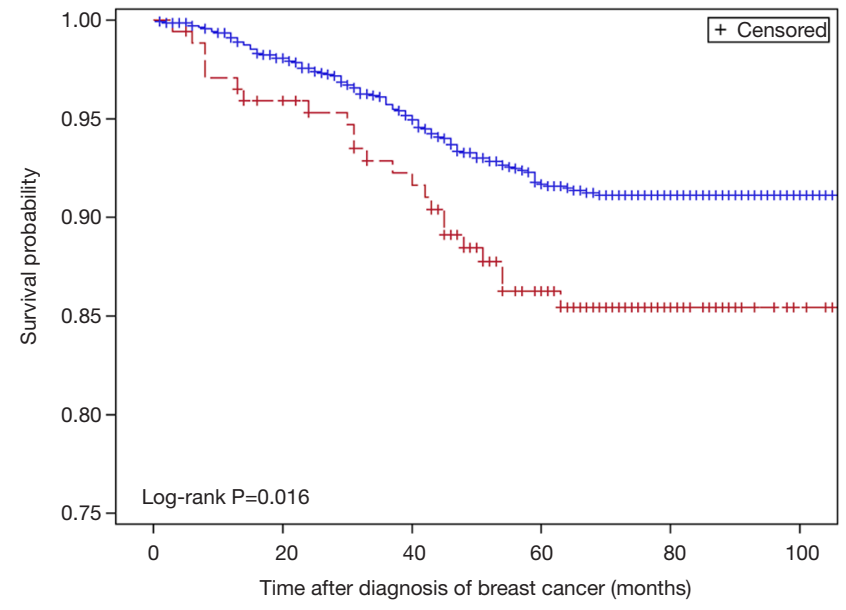

HBsAg-negative 1,375

HBsAg-positive 172
1,309

161
1,229

920

356

B Hepatic metastasis-free survival with number of subjects at risk

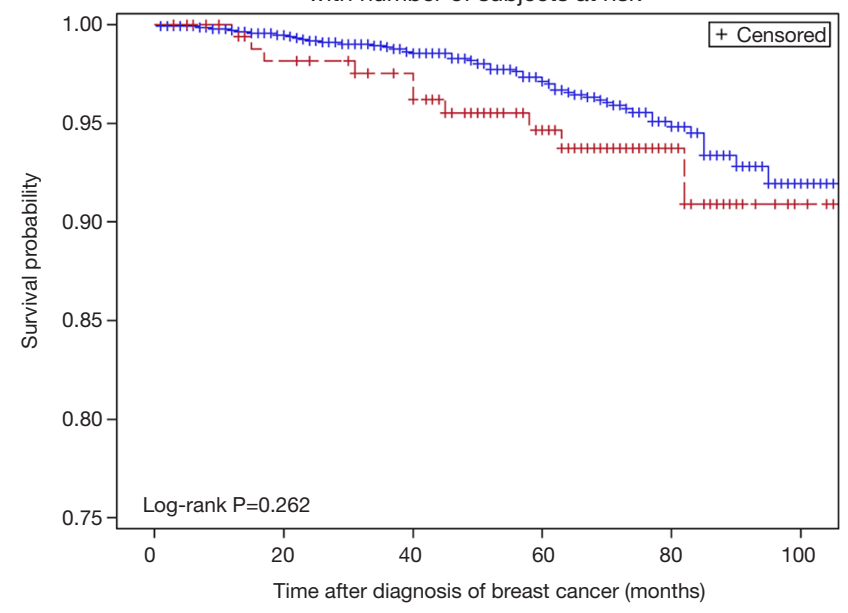

HBsAg-negative 1,375

HBsAg-positive 172

$\begin{array}{ccccc}1,306 & 1,226 & 918 & 354 & 51 \\ 159 & 148 & 108 & 40 & 7\end{array}$

C

Overall survival with number of subjects at risk

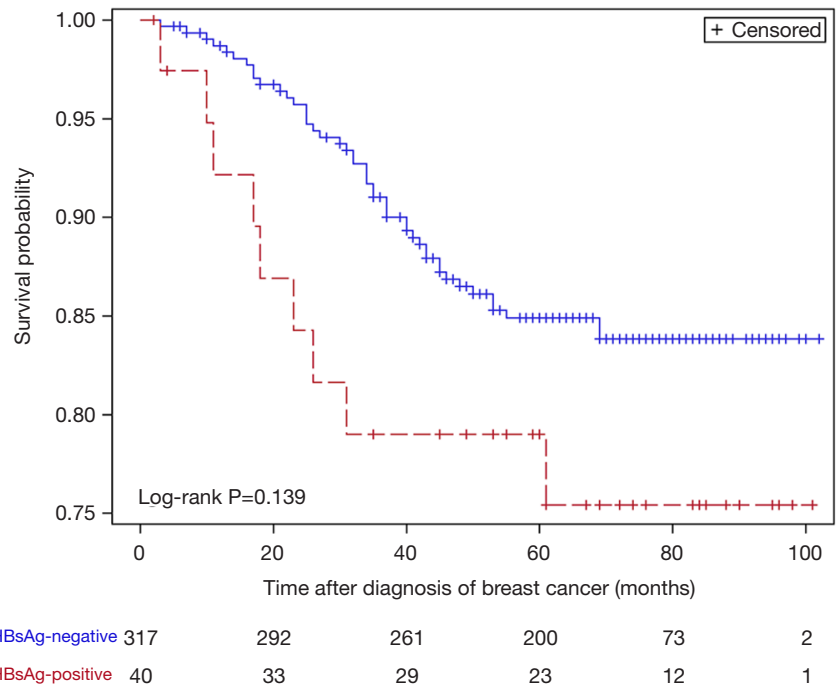

D

D Hepatic metastasis-free survival

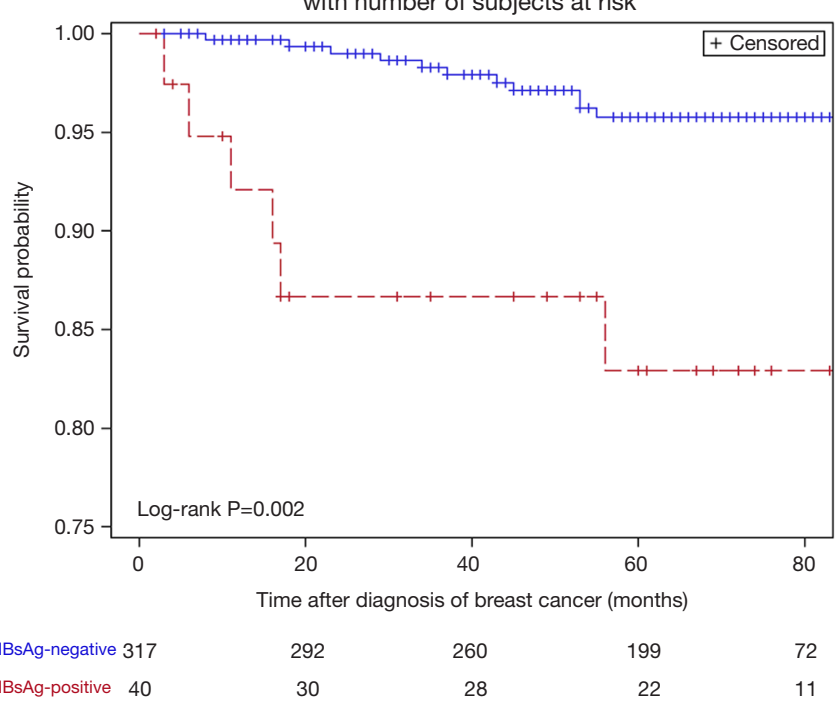

Figure 2 Kaplan-Meier survival curves showing overall survival (A,C) and hepatic metastasis-free survival (B,D) in hepatitis B surface antigen (HBsAg)-positive and -negative luminal breast cancer $(\mathrm{A}, \mathrm{B})$ or non-luminal breast cancer $(\mathrm{C}, \mathrm{D})$. P values were calculated using an unadjusted log-rank test.

was significantly associated with an increased rate of simultaneous liver metastases (11).

Because the liver is most affected by HBV infection, does persistent $\mathrm{HBV}$ infection cause a microenvironment that is prone to liver metastasis? For the effect of chronic HBV infection on liver metastasis, the inconsistent conclusions have been reported in different tumors. It has been reported that chronic HBV infection increased the rate of simultaneous liver metastases in patients with pancreatic cancer but decreases the risk of liver metastasis in colorectal cancer $(11,18)$. Although we found that 5 -year HMFS (93.2\% vs. $97.3 \%, \mathrm{P}=0.016)$ was significantly worse in patients with chronic $\mathrm{HBV}$ infection than in those without $\mathrm{HBV}$ infection, multivariate analysis failed to confirm 
$A$

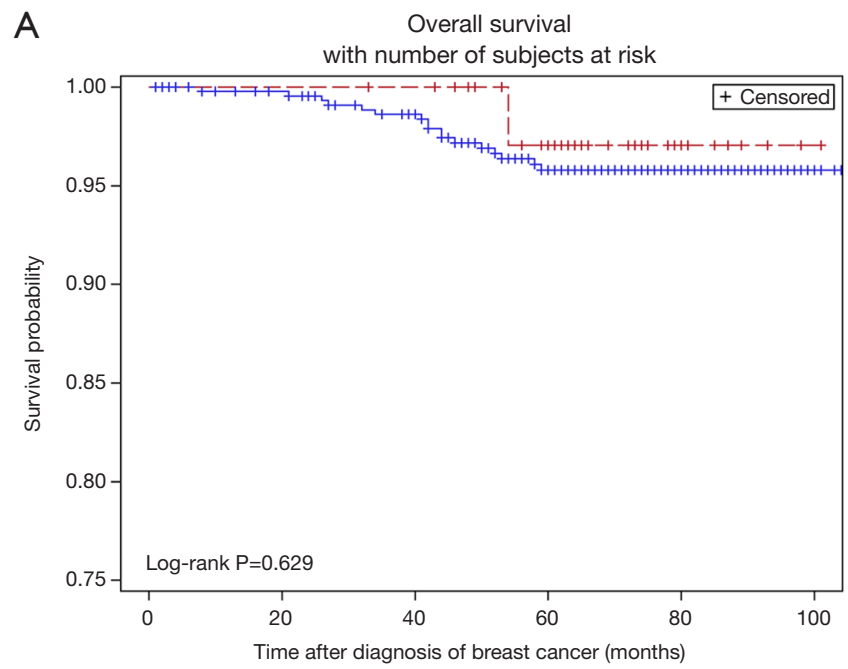

HBsAg-negative 450

HBsAg-positive 41
435

$\begin{array}{cc}417 & 315 \\ 40 & 31\end{array}$
B

B Overall survival

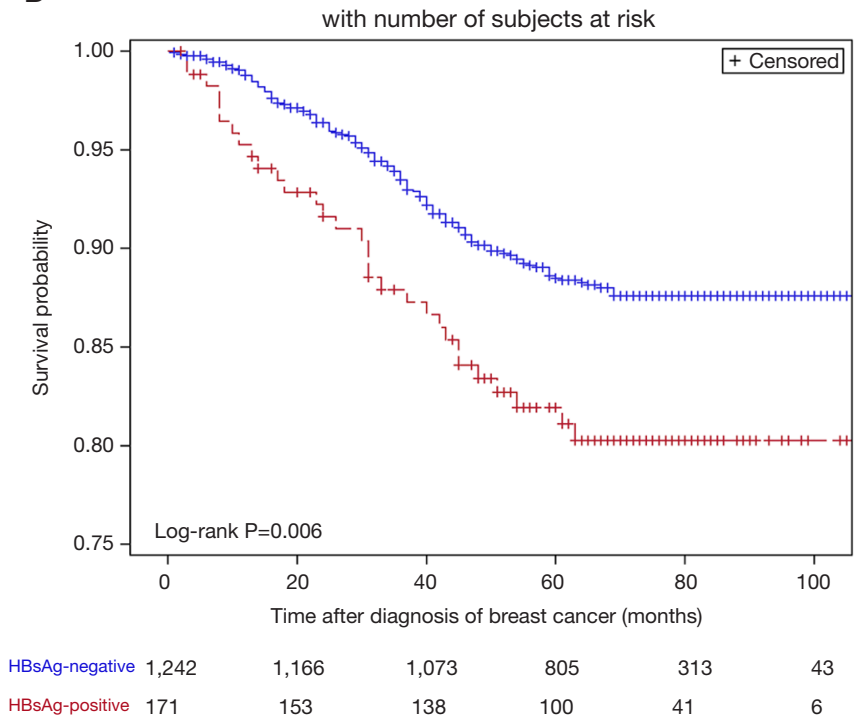

D

D Hepatic metastasis-free survival

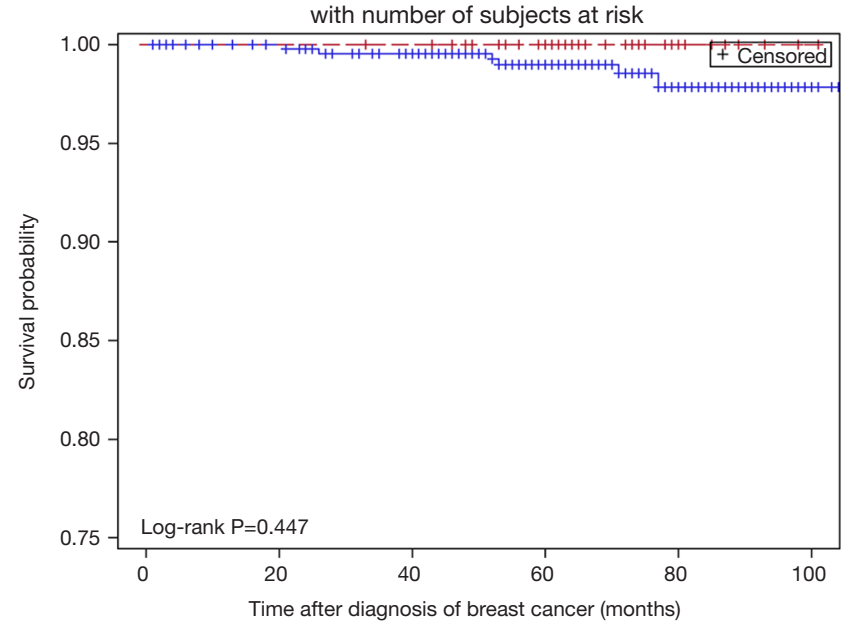

HBsAg-negative 450

HBsAg-positive 41
435

417

40

HBsAg-positive 171

$148 \quad 136$

99

40

6

Figure 3 Kaplan-Meier survival curves showing overall survival (A,B) and hepatic metastasis-free survival (C,D) in hepatitis B surface antigen (HBsAg)-positive and -negative stage I breast cancer (A,D) or stage II/III breast cancer (B,C). P values were calculated using an unadjusted log-rank test.

that chronic HBV infection independently affects HMFS. Therefore, whether HBV infection affects the occurrence of BC liver metastasis needs further research to verify.

The biological mechanisms by which chronic HBV infections affect BC prognosis observed in this study are still elusive. First of all, chronic HBV infection can damage liver cells and impair the deactivation of estrogen by hepatocytes $(8,19)$. Persistent and long-term HBV infection in the liver impairs the normal function of the liver, which leads to elevated estrogen levels as it is primarily inactivated in the liver (20). This may explain to some extent that the observation of chronic HBV infection in this study mainly affects the prognosis of luminal BC, rather than other subtypes. Secondly, HBV may also directly affect breast 
Table 3 Multivariate analysis of prognostic factors in stage II/III breast cancer patients $(\mathrm{N}=1,413)$

\begin{tabular}{|c|c|c|c|c|c|c|c|}
\hline Characteristics & $\mathrm{N}$ & \multicolumn{3}{|c|}{ Univariate analysis } & \multicolumn{3}{|c|}{ Multivariate analysis } \\
\hline \multicolumn{8}{|l|}{ Age at diagnosis } \\
\hline$\leq 35$ years & 136 & 1.00 & - & - & - & - & - \\
\hline$>35$ years & 1,277 & 0.73 & $0.46-1.16$ & 0.180 & - & - & - \\
\hline Negative & 970 & 1.00 & - & - & 1.00 & - & - \\
\hline Positive & 443 & 0.55 & $0.41-0.74$ & $<0.001$ & 0.71 & $0.48-1.03$ & 0.074 \\
\hline \multicolumn{8}{|l|}{ PR } \\
\hline Negative & 448 & 1.00 & - & - & 1.00 & - & - \\
\hline Negative & 1,025 & 1.00 & - & - & 1.00 & - & - \\
\hline Positive & 300 & 1.52 & $1.08-2.14$ & 0.017 & 1.06 & $0.74-1.53$ & 0.741 \\
\hline \multicolumn{8}{|c|}{ Lymphovascular invasion } \\
\hline No & 1,363 & 1.00 & - & - & 1.00 & - & - \\
\hline Yes & 50 & 3.45 & $2.06-5.78$ & $<0.001$ & 1.97 & $1.15-3.39$ & 0.013 \\
\hline \multicolumn{8}{|l|}{ Tumor size } \\
\hline $\mathrm{T} 1$ & 262 & 1.00 & - & - & 1.00 & - & - \\
\hline $\mathrm{T} 2$ & 971 & 0.85 & $0.56-1.29$ & 0.448 & 1.17 & $0.74-1.84$ & 0.511 \\
\hline $\mathrm{N} 2$ & 271 & 3.49 & $2.14-5.70$ & $<0.001$ & 2.85 & $1.69-4.83$ & $<0.001$ \\
\hline N3 & 185 & 7.52 & $4.70-12.00$ & $<0.001$ & 6.34 & $3.78-10.60$ & $<0.001$ \\
\hline \multicolumn{8}{|c|}{ Menopause at diagnosis } \\
\hline No & 531 & 1.00 & - & - & 1.00 & - & - \\
\hline Yes & 882 & 1.40 & $1.03-1.89$ & 0.03 & 1.29 & $0.93-1.78$ & 0.129 \\
\hline \multicolumn{8}{|c|}{ Histological grade } \\
\hline G1 or G2 & 1,012 & 1.00 & - & - & 1.00 & - & - \\
\hline G3 & 401 & 1.72 & $1.26-2.34$ & 0.001 & 1.41 & $1.01-1.95$ & 0.043 \\
\hline \multicolumn{8}{|l|}{ HBsAg status } \\
\hline Negative & 1,242 & 1.00 & - & - & 1.00 & - & - \\
\hline Positive & 171 & 1.72 & $1.17-2.54$ & 0.006 & 1.59 & $1.06-2.39$ & 0.025 \\
\hline
\end{tabular}

$\mathrm{HR}$, hazard ratio; $\mathrm{Cl}$, confidence interval; ER, estrogen receptor; PR, progesterone receptor; HBsAg, hepatitis B surface antigen. 
cells through the action of oncoprotein HBV X protein (HBX) (21-23). For example, several studies have found that BC tissue highly expresses the oncoprotein HBXIP, a protein that interacts with HBX (24). Besides, chronic HBV infection may affect the host's immune function, and it is reported that $\mathrm{HBV}$ is associated with immune dysfunction (25). The results of $\mathrm{Li}$ et al. revealed an HBVinduced immunosuppressive cascade in which $\mathrm{HBV}$ produces inhibitory monocytes that initiate regulatory NK cell differentiation leading to $T$ cell suppression (26). Additionally, patients with chronic or regressive HBV infection are prone to complications of $\mathrm{HBV}$ reactivation during systemic therapy due to the immunosuppressive effects of administered chemotherapy. This may lead to liver damage, which may destroy the effect of anticancer treatment and affect the prognosis of patients $(14,27)$. Most anti-cancer therapies, such as chemotherapy and radiation therapy, can cause immunosuppression, which can cause HBV reactivation and affect treatment $(28,29)$. This may explain in part why the prognosis of patients with stage II/ III complicated with chronic HBV infection is worse, as patients with stage II/III BC tend to receive chemotherapy, which may be harmful to the patient's immune function. Lei et al. found that postoperative HBV reactivation is associated with increased postoperative complications and reduced survival in intrahepatic cholangiocarcinoma (30).

The results of this study provide the first evidence to be known as the poor prognosis of chronic HBV infection in patients with BC. Especially in areas with endemic chronic HBV infection, we should consider the impact of chronic $\mathrm{HBV}$ infection on the prognosis of patients with BC. We recommend that every $\mathrm{BC}$ patient in the $\mathrm{HBV}$ endemic area should have a serological test for HBV at the time of first admission and during the response assessment, whereas patients with serological HBsAg-positive should pay special attention to their possible adverse clinical outcomes. Due to the retrospective nature of this study, we were unable to examine the effect of HBV-DNA levels and antiviral therapy on the prognosis of patients with $\mathrm{BC}$ with chronic $\mathrm{HBV}$ infection. This is a major shortcoming of current research, and therefore, whether BC patients with higher $\mathrm{HBV}$ infection burden have poor survival remains unknown. With the increasing use of chemotherapy, targeted therapy, and endocrine therapy for systemic treatment of BC, the occurrence of $\mathrm{HBV}$ reactivation may increase during this period. However, there is a lack of data on clinical management of $\mathrm{HBV}$ screening and reactivation as well as BC patients with $\mathrm{HBV}$ infection. The difference in the risk of $\mathrm{HBV}$ reactivation in $\mathrm{BC}$ patients during different treatments and how to manage $\mathrm{BC}$ in the $\mathrm{HBV}$ endemic area deserves further study.

\section{Conclusions}

This study proved that chronic HBV infection was an independent risk factor for prognosis in patients with stage II/III BC. It is necessary to further confirm these results through large prospective studies, including the impact of HBV DNA load on $\mathrm{BC}$ prognosis. In addition, there is a need to investigate the underlying mechanisms of chronic $\mathrm{HBV}$ infection affecting the survival outcome of stage II/III BC patients.

\section{Acknowledgments}

We thank the staff of the Medical Records Management Section of the Sun Yat-sen University Cancer Center for supporting the research.

Funding: The study was funded by the National Natural Science Foundation of China (grant numbers: 81672598 , 81772961).

\section{Footnote}

Conflicts of Interest: The authors have no conflicts of interest to declare.

Ethical Statement: The authors are accountable for all aspects of the work in ensuring that questions related to the accuracy or integrity of any part of the work are appropriately investigated and resolved. This study was approved by the Ethics Committee of the Cancer Center of Sun Yat-sen University (No. GZR2016-076). All patients signed the informed consent form.

Open Access Statement: This is an Open Access article distributed in accordance with the Creative Commons Attribution-NonCommercial-NoDerivs 4.0 International License (CC BY-NC-ND 4.0), which permits the noncommercial replication and distribution of the article with the strict proviso that no changes or edits are made and the original work is properly cited (including links to both the formal publication through the relevant DOI and the license). See: https://creativecommons.org/licenses/by-nc-nd/4.0/.

\section{References}

1. Tang LSY, Covert E, Wilson E, et al. Chronic Hepatitis B 
Infection: A Review. JAMA 2018;319:1802-13.

2. Te HS, Jensen DM. Epidemiology of hepatitis B and C viruses: a global overview. Clin Liver Dis 2010;14:121 , vii.

3. Lemaitre M, Brice P, Frigeni M, et al. Hepatitis B virusassociated B-cell non-Hodgkin lymphoma in non-endemic areas in Western Europe: Clinical characteristics and prognosis. J Infect 2020;80:219-24.

4. Mahale P, Engels EA, Koshiol J. Hepatitis B virus infection and the risk of cancer in the elderly US population. Int J Cancer 2019;144:431-9.

5. Clements O, Eliahoo J, Kim JU, et al. Risk factors for intrahepatic and extrahepatic cholangiocarcinoma: A systematic review and meta-analysis. J Hepatol 2020;72:95-103.

6. Yang F, Ma L, Yang Y, et al. Contribution of Hepatitis B Virus Infection to the Aggressiveness of Primary Liver Cancer: A Clinical Epidemiological Study in Eastern China. Front Oncol 2019;9:370.

7. Liang JH, Gao R, Dai JC, et al. The prognostic role of $\mathrm{HBV}$ infection in chronic lymphocytic leukemia. J Cancer Res Clin Oncol 2018;144:1309-15.

8. Cui H, Jin Y, Chen F, et al. Clinicopathological evidence of hepatitis $\mathrm{B}$ virus infection in the development of gastric adenocarcinoma. J Med Virol 2020;92:71-7.

9. Weng JJ, Wei JZ, Li M, et al. Effects of hepatitis B virus infection and antiviral therapy on the clinical prognosis of nasopharyngeal carcinoma. Cancer Med 2020;9:541-51.

10. Desai R, Patel U, Sharma S, et al. Association Between Hepatitis B Infection and Pancreatic Cancer: A Population-Based Analysis in the United States. Pancreas 2018;47:849-55.

11. Wei XL, Qiu MZ, Chen WW, et al. The status of HBV infection influences metastatic pattern and survival in Chinese patients with pancreatic cancer. J Transl Med 2013;11:249.

12. Zhang X, Zhou Y, Chen C, et al. Hepatitis B virus reactivation in cancer patients with positive Hepatitis B surface antigen undergoing PD-1 inhibition. J Immunother Cancer 2019;7:322.

13. Yao ZH, Liao WY, Ho CC, et al. Incidence of hepatitis $B$ reactivation during epidermal growth factor receptor tyrosine kinase inhibitor treatment in non-small-cell lung cancer patients. Eur J Cancer 2019;117:107-15.

14. Yang HC, Tsou HH, Pei SN, et al. Quantification of HBV core antibodies may help predict HBV reactivation in patients with lymphoma and resolved $\mathrm{HBV}$ infection. J
Hepatol 2018;69:286-92.

15. Kusumoto S, Arcaini L, Hong X, et al. Risk of HBV reactivation in patients with $\mathrm{B}$-cell lymphomas receiving obinutuzumab or rituximab immunochemotherapy. Blood 2019;133:137-46.

16. Wang F, Xu RH, Luo HY, et al. Clinical and prognostic analysis of hepatitis B virus infection in diffuse large B-cell lymphoma. BMC Cancer 2008;8:115.

17. Liu X, Li X, Jiang N, et al. Prognostic value of chronic hepatitis $\mathrm{B}$ virus infection in patients with nasopharyngeal carcinoma: analysis of 1301 patients from an endemic area in China. Cancer 2014;120:68-76.

18. Qiu HB, Zhang LY, Zeng ZL, et al. HBV infection decreases risk of liver metastasis in patients with colorectal cancer: A cohort study. World J Gastroenterol 2011;17:804-8.

19. Jiang M, Klein M, Zanger UM, et al. Inflammatory regulation of steroid sulfatase: A novel mechanism to control estrogen homeostasis and inflammation in chronic liver disease. J Hepatol 2016;64:44-52.

20. Raimondo G, Caccamo G, Filomia R, et al. Occult HBV infection. Semin Immunopathol 2013;35:39-52.

21. Adhikari VP, Lu LJ, Kong LQ. Does hepatitis B virus infection cause breast cancer. Chin Clin Oncol $2016 ; 5: 81$

22. Liu BW, Wang TJ, Li LL, et al. Oncoprotein HBXIP induces $\mathrm{PKM} 2$ via transcription factor E2F1 to promote cell proliferation in ER-positive breast cancer. Acta Pharmacol Sin 2019;40:530-8.

23. Liu B, Wang T, Wang H, et al. Oncoprotein HBXIP enhances HOXB13 acetylation and co-activates HOXB13 to confer tamoxifen resistance in breast cancer. J Hematol Oncol 2018;11:26.

24. Zhou XL, Zhu CY, Wu ZG, et al. The oncoprotein HBXIP competitively binds KEAP1 to activate NRF2 and enhance breast cancer cell growth and metastasis. Oncogene 2019;38:4028-46.

25. Park JJ, Wong DK, Wahed AS, et al. Hepatitis B Virus-Specific and Global T-Cell Dysfunction in Chronic Hepatitis B. Gastroenterology 2016;150:684-95.e5.

26. Li H, Zhai N, Wang Z, et al. Regulatory NK cells mediated between immunosuppressive monocytes and dysfunctional T cells in chronic HBV infection. Gut 2018;67:2035-44.

27. Loomba R, Liang TJ. Hepatitis B Reactivation Associated With Immune Suppressive and Biological Modifier Therapies: Current Concepts, Management Strategies, and Future Directions. Gastroenterology 
2017;152:1297-309.

28. Lv JW, Chen YP, Huang XD, et al. Hepatitis B virus screening and reactivation and management of patients with nasopharyngeal carcinoma: A large-scale, big-data intelligence platform-based analysis from an endemic area. Cancer 2017;123:3540-9.

29. Mücke MM, Backus LI, Mücke VT, et al. Hepatitis B virus reactivation during direct-acting antiviral therapy for hepatitis C: a systematic review and meta-analysis. Lancet Gastroenterol Hepatol 2018;3:172-80.

30. Lei Z, Xia Y, Si A, et al. Antiviral therapy improves survival in patients with $\mathrm{HBV}$ infection and intrahepatic cholangiocarcinoma undergoing liver resection. J Hepatol 2018;68:655-62.

Cite this article as: Xiao W, Zhou Y, Yu P, Yang A, Zheng S, Tang H, Xie X. Prognostic value of chronic hepatitis B virus infection in patients with breast cancer in a hepatitis B virus endemic area. Ann Transl Med 2020;8(5):180. doi: 10.21037/ atm.2020.01.97 